\title{
Replication-defective recombinant Semliki Forest virus encoding GM-CSF as a vector system for rapid and facile generation of autologous human tumor cell vaccines
}

\author{
S Withoff ${ }^{1, *}$, KL Glazenburg, ${ }^{1, *}$, ML van Veen ${ }^{1}$, MMJ Kraak ${ }^{1}$, GAP Hospers ${ }^{2}$, S Störkel ${ }^{3}$, \\ EGE de Vries $^{2}$, J Wilschut ${ }^{1}$ and T Daemen ${ }^{1}$ \\ ${ }^{1}$ Department of Medical Microbiology, Molecular Virology Section, and ${ }^{2}$ Department of Medical Oncology, University of Groningen \\ and Academic Hospital, Groningen, The Netherlands; and ${ }^{3}$ Institut für Pathologie, Universität Witten/Herdecke, Wuppertal, \\ Germany
}

\begin{abstract}
This paper describes the production of recombinant Semliki Forest virus encoding murine or human granulocyte-macrophage colony-stimulating factor (GM-CSF) and the capacity of these vectors to transduce murine and human tumor cells ex vivo. High-titer stocks (up to $3 \times 10^{9}$ particles $/ \mathrm{ml}$ ) of conditionally infective, replication-defective, recombinant SFV particles were generated using the SFV Helper-2 system. It is shown that the recombinant SFV/GM-CSF virus, as well as recombinant SFV carrying the $\beta$-galactosidase reporter gene, efficiently transduce both murine tumor cell lines as well as primary human renal carcinoma cells. Using ELISA's specific for GM-CSF, levels of GM-CSF production by the cells were determined. Levels of murine GM-CSF (mGM-
\end{abstract}

CSF) produced by SFV/mGM-CSF transduced renal cell cancer cultures were equal to or higher than corresponding levels reported in the literature after transduction of similar renal carcinoma cell cultures using a retroviral vector system. The biological activity of GM-CSF was demonstrated by using cells which are dependent on GM-CSF for growth and by using primary bone marrow cells. All the transduced cell cultures (including the human renal cell carcinoma samples) produced GM-CSF for up to at least 4 days after transduction. The results imply that the recombinant SFV system can be used for rapid and facile preparation of autologous cancer cell vaccines. Gene Therapy (2001) 8, 1515-1523.

Keywords: Semliki Forest virus; autologous tumor cell vaccine; GM-CSF; cytokine gene transfer; gene therapy

\section{Introduction}

The concept of immune surveillance of malignant cell formation and proliferation is currently widely accepted. According to this theory, aberrant cells generated through spontaneous or induced mutagenesis will be eliminated. However, malignant cells may develop mechanisms by which immune surveillance is evaded and tumor outgrowth may occur. These mechanisms include down-regulation of MHC molecules, adhesion molecules and/or costimulatory molecules resulting in a considerably reduced immunogenicity of the tumor.

Tumor cell vaccine approaches aim at enhancing the immunogenicity of (autologous) tumor cells. Specifically, by introducing genes that encode immunomodulators into tumor cells, these cells protractedly express and secrete growth factors. It is anticipated that, upon readministration of the cells to the patient, antigen-presenting cells (APCs), such as macrophages and dendritic cells,

Correspondence: Dr KL Glazenburg, Department of Medical Microbiology, Molecular Virology Section, University of Groningen, Ant. Deusinglaan 1, 9713 AV Groningen, The Netherlands

*SW and KLG contributed equally to this study

Received 30 January 2001; accepted 18 July 2001 will be attracted to the site of injection and will become activated. Possibilities that are already explored in the clinic involve immunization with autologous tumor cells, genetically modified with recombinant viral vectors to produce interferon-gamma, IL-4, IL-12, and GM-CSF. ${ }^{1-6}$ At present, GM-CSF appears to be the most efficient cytokine for obtaining optimal immune responses. ${ }^{7}$

Within the context of the use of cytokine gene-modified autologous tumor cell vaccines, currently, in the clinic retrovirus vectors are applied most widely for delivery of the cytokine gene to the tumor cells, ${ }^{7-11}$ although other viral delivery systems are also under investigation. ${ }^{12-14}$ Yet, a major drawback of retroviral gene delivery is that the target cell needs to be competent of division, since only dividing cells are susceptible to retroviral gene integration. In most human solid tumors the greater part of the resection will, however, consist of nondividing cells. In addition, integration of the viral genome is not required for short-term cytokine expression and is associated with risks of insertional mutagenesis. Furthermore, retroviral stocks of high titers are difficult to produce, and transduction efficiencies are low, which often necessitates clonal selection.

In this study, we used a replication-defective recombinant Semliki Forest virus (SFV) system, developed by 
Liljeström and coworkers, for introduction of the GMCSF gene into tumor cells. ${ }^{15-18} \mathrm{SFV}$ is a positive-strand RNA virus belonging to the genus Alphaviruses of the family Togaviridae. ${ }^{19}$ Alphaviruses replicate in the host cell cytosol without integration of the viral genetic material in the cellular genome. Thus, alphaviruses efficiently infect nondividing cells. Furthermore, alphaviruses, including SFV, have a very broad host cell specificity. ${ }^{19}$ Cells infected with either native or replication-defective recombinant alphaviruses die by apoptosis ${ }^{20,21}$ ensuring that the foreign genetic material does not persist within the organism. These characteristics make alphavirus-based vectors very promising systems for use in gene therapy approaches where transient gene expression is desirable. These applications include genetic immunization strategies. SFV vectors have been used by us and by others to elicit both humoral and cellular immune responses in mice against human papillomavirus, ${ }^{22}$ influenza, ${ }^{23}$ louping ill virus ${ }^{24}$ and mastocytoma P815 tumor antigens. ${ }^{25}$ In primates it has been shown that SFV vectors encoding envelope glycoproteins of simian immunodeficiency virus (SIV) could protect monkeys against a lethal SIV challenge ${ }^{26}$ and that human immunodeficiency virus-1 (HIV-1) envelope vaccines (based on SFV) induced $\mathrm{B}$ and $\mathrm{T}$ cell responses and modified challenge-virus replication. ${ }^{27}$

The SFV expression system is based on the availability of an infectious clone of the virus. RNA transcripts produced in vitro from this clone (plasmid pSP6-SFV4, containing a cDNA copy of the full-length viral genome), direct virus replication when introduced into cells by, for example, electroporation. To permit insertion of heterologous genes, the subgenomic region of the SFV genome, encoding the viral structural proteins, has been deleted from pSP6-SFV4 and replaced by a polylinker site to produce the vector pSFV1. ${ }^{16}$ This vector retains the nonstructural region of the viral genome encoding the RNA replicase, thus preserving the self-replicating ability of the $\mathrm{SFV}$ replicon. Insertion of a heterologous gene in the polylinker site of SFV1 now allows in vitro production of RNA transcripts containing the foreign gene in the context of the SFV replicon. When introduced into cells these RNA transcripts will direct extensive synthesis of the heterologous protein with simultaneous down-regulation of host-cell protein synthesis. ${ }^{16}$ Packaging of the recombinant RNA containing the heterologous gene into SFV particles can be achieved by the introduction of two independent RNAs into cells. The first RNA contains the replicase, the subgenomic promotor and the heterologous gene, and the second RNA (the 'helper') contains the subgenomic promotor and the genes encoding the viral structural proteins. These RNAs will direct the production of recombinant SFV particles in producer cells. Importantly, these particles only package the recombinant RNA containing the heterologous gene as only this RNA carries the RNA packaging signal located on the nonstructural region of the viral genome. Since the structural genetic information is not packaged into particles, the recombinant virus is replication-defective, and thus gives rise to only a single round of target cell infection.

Recently, we showed that intraperitoneal (i.p.) injection of mice with commercially available recombinant GMCSF expanded the population of peritoneal macrophages. These macrophages could be activated to a high level of cytotoxicity. ${ }^{28}$ Recently, we also used recombinant SFV particles for in vivo and in vitro transfection of peritoneal cells. It was demonstrated that i.p. injection of GM-CSF particles results in high-level expression of the recombinant protein in the peritoneal cavity and macrophage activation. Treatment of tumor-bearing mice resulted in an inhibition of tumor growth. ${ }^{29}$ To induce tumor-specific immune responses we now focus on the ex vivo transduction of tumor cells to develop a cytokine-secreting autologous tumor vaccine. The paper describes the production of recombinant SFV particles carrying the murine or human GM-CSF gene (mGM-CSF and hGM-CSF, respectively). We determined the susceptibility of a broad set of cell types for SFV. In addition, recombinant protein expression was evaluated quantitatively and qualitatively in cell lines and in primary cultures of human renal cell carcinoma.

\section{Results}

\section{Production of recombinant SFV particles}

Recombinant SFV particles were produced by electroporating two in vitro synthesized subgenomic RNAs into BHK cells. One subgenomic RNA encoded the viral RNA replicase, the cloned transgene (mGM-CSF, hGM-CSF, or reporter gene $L a c Z$ ), and contained the viral packaging signal. Hence, this RNA was incorporated into viral particles. The other subgenomic RNA lacked the packaging signal and encoded the structural proteins with a mutation in the p62 gene. ${ }^{15}$ The mutation in p62 prevents cleavage of precursor p62 into E2 and E3. Therefore, generated stocks of virus contained non-infectious virions with precursor protein p62 in the envelope. Immediately before transduction, particles were activated by cleaving the precursor with chymotrypsin that liberated the fusion active E2 protein. $^{15}$

Twenty-four hours after electroporating RNA into BHK cells, particles were harvested. Using the procedures described in Materials and methods, SFV particle-stocks were obtained with titers ranging from 2 $\times 10^{8}$ to $3 \times 10^{9}$ infectious $\mathrm{U} / \mathrm{ml}$. The total amount of particles yielded per electroporation varied from $2 \times 10^{9}$ to $3 \times 10^{10}$ infectious units. Virus particles were named $\mathrm{SFV} / \mathrm{mGM}-\mathrm{CSF}, \mathrm{SFV} / \mathrm{hGM}-\mathrm{CSF}, \mathrm{SFV} / \mathrm{LacZ}$ and SFV/'empty'. Titers of SFV/LacZ particles either as determined by nsP3 staining or as determined by $\beta$ galactosidase activity were fully comparable (results not shown).

\section{Transducibility of cell lines}

To determine the susceptibility of different cell types for SFV we transduced the cell lines BHK-21, Vero, B16F10, C26, BLK, TC-1, and RT112 cells with SFV/LacZ particles (Table 1). BHK cells, which were used to produce SFV particles, are highly susceptible to SFV transduction and were used as standard to determine virus titers. The susceptibility of Vero cells and TC-1 cells was comparable to that of BHK cells. B16 cells and C26 cells were less susceptible. Transduction of B16 cells and C26 cells with an MOI of 1 resulted in approximately 1\% positive cells. Transduction with higher MOIs increased the number of positive cells. Transduction with a MOI of 500 resulted in 5\% positive B16 and C26 cells. BLK and RT112 cells were intermediately susceptible. Transduction of BLK cells with a MOI of 1 resulted in $15 \%$ positive cells and 
Table 1 Susceptibility of several cell lines for SFV-mediated transduction

\begin{tabular}{lccccccc}
\hline Cell type & BHK-21 & VERO & B16F10 & C26 & BLK & TC-1 & RT-112 \\
\hline $\begin{array}{l}\text { Transducibility } \\
\text { (pos. cells/100 IUs) }\end{array}$ & 100 & 104 & 1 & 1 & 15 & 100 & 8.5 \\
\hline
\end{tabular}

100 infectious units of marker virus SFV/LacZ were used to transduce confluent monolayers of cells. The number of LacZ-positive cells (mean of two wells) are shown.

transduction of the human bladder tumor cell RT112 resulted in $8.5 \%$ positive cells.

\section{Transduction of BHK-21 and B16F10 cells with SFV/GM-CSF (mouse and human) and kinetics of GM- CSF secretion}

In Figure 1a, mGM-CSF secretion by BHK-21 cells in time is shown for three different transduction experiments. A MOI of 50 was used in all three cases. The amount of mGM-CSF in virus stocks which was synthesized during virus production in BHK-21 cells was less than $0.1 \mathrm{ng} / 10^{6}$ particles and therefore did not significantly contribute to mGM-CSF production measured. In Figure $1 b$ and $c$ it is shown that despite the low transducibility of B16F10 and C26 cells compared with BHK-21 cells, mGM-CSF expression could still be measured. In Figure 1d the production of hGM-CSF in BHK-21 cells transduced with $\mathrm{SFV} / \mathrm{hGM}-\mathrm{CSF}$ is shown. Compared with the production of mGM-CSF, the production of hGM-CSF was approximately five times less. The MOI of 50 and 5 only slightly influenced the amount of GM-CSF produced. Decreasing the $\mathrm{MOI}$ to 0.5 resulted in approximately $50 \%$ reduction of GM-CSF production. The presence of fetal calf serum, which will have to be absent when generating virus for vaccination purposes, was not required for efficient GMCSF production (Figure 1d).
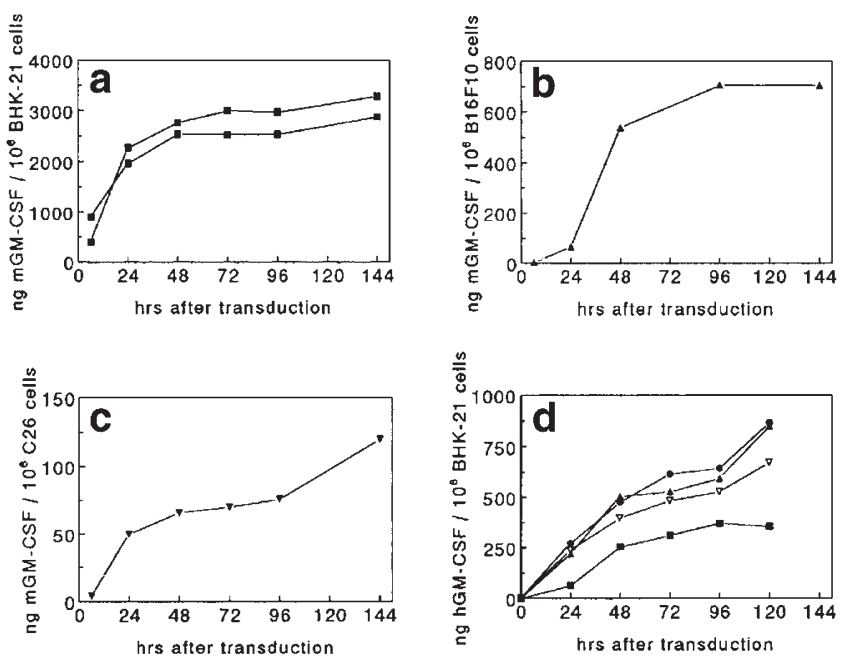

Figure 1 (a) BHK-21 cells were transduced with SFV/mGM-CSF particles at $M O I=50(n=2 ; \boldsymbol{\square})$. Cytokine production was measured using a $m G M$-CSF-specific ELISA. ( $b$ and c) $m G M$-CSF expression levels by B16F10 cells $(\boldsymbol{\Delta})$ and C26 cells $(\boldsymbol{\nabla})$ respectively. (d) BHK-21 cells transduced with SFV/hGM-CSF particles at MOIs of 50, 5, and $0.5(\mathbf{0}, \nabla, \mathbf{\square})$ and at MOI of 5 without serum $(\mathbf{\Delta})$.

\section{Determination of the biological activity of GM-CSF produced by BHK-21 cells}

Sequencing of $\mathrm{pSFV} 1 / \mathrm{mGM}-\mathrm{CSF}$ had shown that a point mutation in the mGM-CSF gene was present: we therefore tested whether the mGM-CSF produced by recombinant SFV/mGM-CSF particles is biologically active. A dilution series of commercial mGM-CSF (produced in Escherichia coli) and of medium containing mGM-CSF obtained from SFV/mGM-CSF transduced BHK-21 cells was prepared. These preparations were tested using the FDCP-1 cell proliferation assay. In Figure 2 the results of representative experiments show that the biological activities of the SFV/mGM-CSF encoded mGM-CSF and the commercial available mGM-CSF are identical. This observation was confirmed using a colony-forming assay with mouse bone marrow cells (results not shown). The concentration curves shown in Figure 2 are in accordance with concentrations described to be needed for optimal stimulation of the FDCP-1 cell line $\left(200-800 \mathrm{ng} / \mathrm{ml}^{30}\right)$. Freezing/thawing or incubation for 3 days at $37^{\circ} \mathrm{C}, 5 \%$ $\mathrm{CO}_{2}$ (not shown) did not affect the results indicating that mGM-CSF produced was as stable as commercially available mGM-CSF.

To examine the biological activity of hGM-CSF, TF-1 cells were used. These cells were cultured for several passages with $10 \mathrm{ng} / \mathrm{ml} \mathrm{hGM}-\mathrm{CSF}$ produced by SFV/hGM-CSF transduced BHK-21 cells, with $10 \mathrm{ng} / \mathrm{ml}$ of recombinant hGM-CSF, or without hGM-CSF. The proliferation of the cells, as determined by cell counting, clearly demonstrated that cell growth was supported

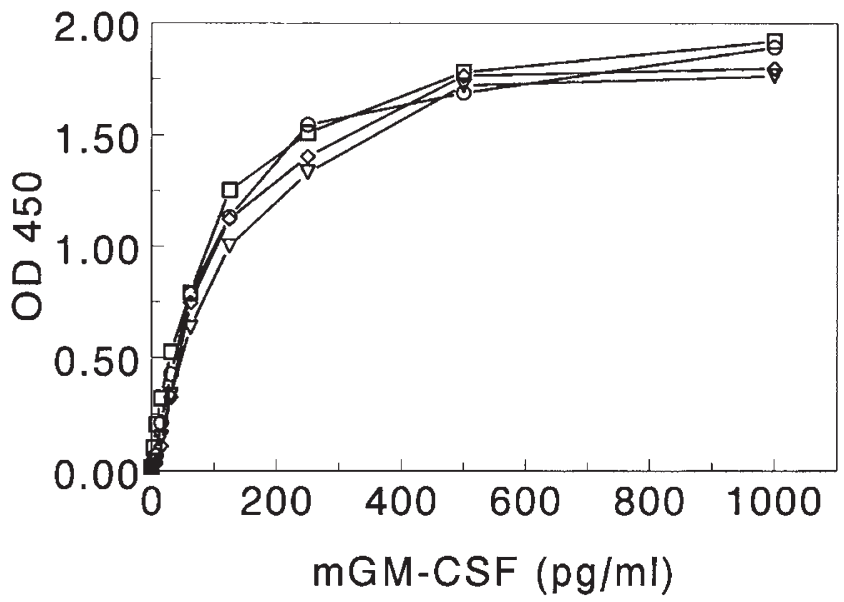

Figure 2 Bioactivity of $m G M-C S F$ based on an FDCP-1 proliferation assay. The bioactivity of two different samples of medium of SFV/mGMCSF transduced BHK-21 cells $(0, \nabla)$, commercially available mGM-CSF ( $\square$ ) and medium of SFV/mGM-CSF transduced BHK-21 cells that was frozen at $-20^{\circ} \mathrm{C}$ and thawed $(\diamond)$ were compared. 
equally well by hGM-CSF produced by BHK-21 as by recombinant hGM-CSF (Figure 3). In addition, human bone marrow cells were used to determine the biological activity of hGM-CSF: $10^{5}$ bone marrow cells were incubated with either a standard control mixture of recombinant hGM-CSF (10 ng/ml) and rhIL-3 (10 ng/ml), BHK21 produced hGM-CSF (10 ng/ml), or without hGM-CSF. The number of colonies formed after stimulation with BHK-21 produced hGM-CSF were comparable to the number produced after stimulation with the recombinant hGM-CSF/IL-3 mix (74 versus 70, respectively). Without hGM-CSF only 14 colonies were counted.

\section{Transduction of primary cultures of human renal cell carcinomas and accompanying healthy kidney tissue samples}

In Figure 4 and Table 2 results obtained with human tumor material are shown. All cultures transduced with $\mathrm{SFV} / \mathrm{mGM}-\mathrm{CSF}$ expressed mGM-CSF (Figure 4). Expression levels at $24 \mathrm{~h}$ after transduction were higher or comparable to hGM-CSF expression levels found after transduction of RCC samples using recombinant retrovirus. ${ }^{1,8}$

In most cases expression levels were higher in the RCC samples than in the healthy tissue of the same patient, however, the opposite was found as well (patient D). When MOIs were increased higher expression levels could be measured. In none of the untransduced samples was mGM-CSF expression measured indicating that the ELISA is specific for mGM-CSF (in Simons et al ${ }^{1}$ it was described that human RCC cells produce autologous hGM-CSF). The different samples produced GM-CSF for up to 4 days after transduction.

We next determined the expression of hGM-CSF by RCC upon transduction with SFV/hGM-CSF. These experiments were performed using primary cells that had been frozen in liquid nitrogen over 1 year. RCC and normal cells were successfully recovered and cultured from cells obtained from patients $\mathrm{A}$ and $\mathrm{F}$. Expression of endogenous hGM-CSF in untransduced cells varied from 1.2 to $7.6 \mathrm{ng} / 10^{6}$ cells $/ 48 \mathrm{~h}$. Transduction of normal and tumor cells with SFV/hGM-CSF resulted in hGM-CSF

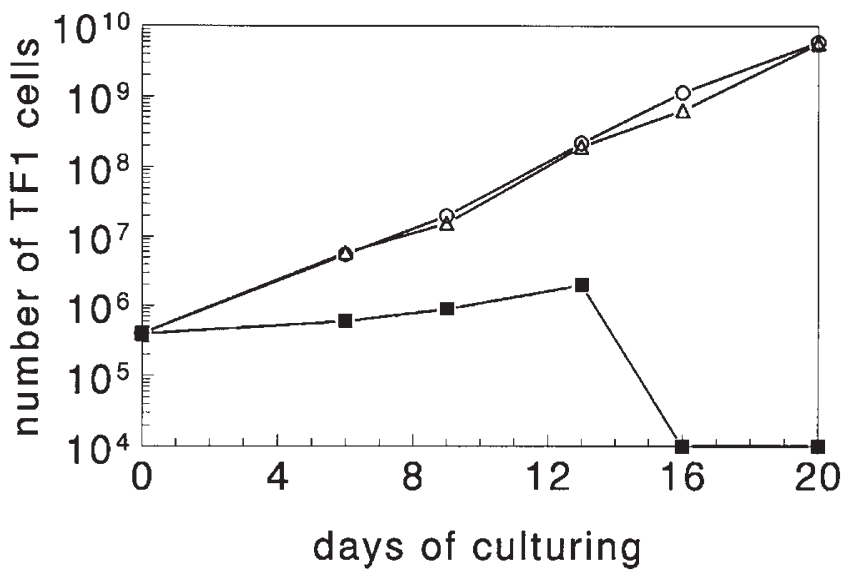

Figure 3 Bioactivity of hGM-CSF using a TF-1 cell proliferation assay. TF-1 cells were cultured in the presence or absence of hGM-CSF. At each passage, cells were counted and split in a 1:5 ratio. Growth of cell cultures supplemented with $10 \mathrm{ng} / \mathrm{ml} h \mathrm{~h}$ GM-CSF of SFV/hGM-CSF origin (O) or $10 \mathrm{ng} / \mathrm{ml}$ of control hGM-CSF $(\triangle)$. Growth of cells without hGM-CSF
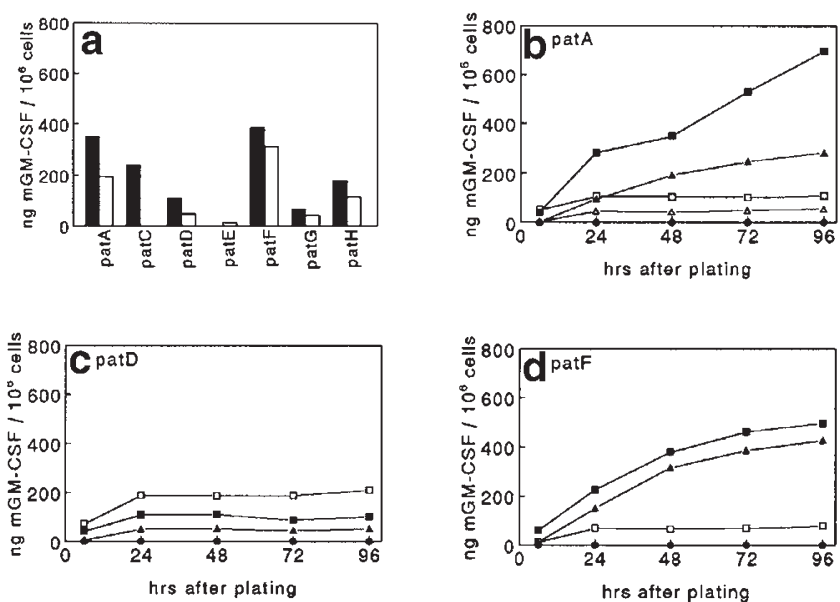

Figure 4 Production of $m G M-C S F$ after transduction of human renal cell carcinomas. In (a) the data for all RCC samples are shown when transduced with SFV/mGM-CSF at MOI 500 (black bars) or MOI 50 (white bars). The bars represent $m G M-C S F$ levels measured 48 h after transduction. In $b, c$ and $d m G M-C S F$ production in time is shown for three patients. Open symbols depict normal tissue, closed symbols malignant tissue samples ( $\square, \mathbf{\square}$ transduced at MOI 500; $\triangle$, $\mathbf{\Delta}$ transduced at MOI 50; $\bigcirc$ not transduced).

levels ranging from 45.2 to $84.8 \mathrm{ng} / 10^{6}$ cells. The production level was higher then the threshold level of 35 $\mathrm{ng} / 10^{6}$ cells required to induce an immune response ${ }^{1,8}$ but was less than that observed after transduction of fresh primary cell cultures transduced with SFV/mGMCSF.

\section{Discussion}

The most widely applied viral vectors in immunotherapy approaches against cancer are recombinant retroviral and adenoviral systems. Retroviral yields (titers) are usually low and in order to transfect a target cell, the vector has to be integrated into the host's genome, requiring active cell division of the host cell. For transfection purposes of tumor material this is a serious drawback as the greater part of the cells in most solid tumors does not divide and the viable cells are difficult to culture. Adenovirus can be produced at high titers and the genetic material remains episomal. This virus can infect dividing and nondividing target cells. Drawbacks of this system are that adenovirus infections naturally occur in humans and an immune reaction to adenoviral proteins cannot be excluded. This makes (multiple) injections with adenovirus or with adenovirus-infected cells a vulnerable approach. Therefore, researchers have set out to investigate characteristics of new viral vectors. New emerging systems are based on adeno-associated virus, Herpes simplex virus and Vaccinia virus or related pox viruses (fowl pox or canary pox virus). ${ }^{31-33}$ To improve tumor antigen presentation, genes were delivered directly into dendritic cells ${ }^{34,35}$ and combinations of different approaches have been suggested. ${ }^{36}$ Despite these new vector developments the retroviral approach for delivery of cytokine genes to autologous tumor cells is thusfar the most widely used in the clinic, also for delivery of GM-CSF. ${ }^{1,4,37}$

In the present study we have studied transgene expression using the recombinant SFV system. The recombinant SFV system has several advantages. High 
Table 2 Expression of hGM-CSF in human RCC

\begin{tabular}{|c|c|c|c|c|c|c|}
\hline \multirow[t]{2}{*}{ Patient } & \multirow[t]{2}{*}{ Kidney tissue } & \multicolumn{5}{|c|}{$h G M-C S F\left(n g / 10^{6}\right.$ cells $)$} \\
\hline & & Baseline level & Transduced exp. 1 & Transduced exp. 2 & Transduced exp. 3 & Mean increase ( \pm s.d.) \\
\hline $\mathrm{A}$ & tumor & 1.2 & 57.2 & 45.2 & ND & $50.0 \pm 8.5$ \\
\hline $\mathrm{A}$ & normal & 7.6 & 59.6 & 55.6 & 64.8 & $52.4 \pm 4.6$ \\
\hline $\mathrm{F}$ & tumor & 3.6 & 60.8 & 84.8 & 48.4 & $61.1 \pm 18.5$ \\
\hline $\mathrm{F}$ & normal & 6.6 & 66.4 & 63.6 & 57.6 & $55.9 \pm 4.5$ \\
\hline
\end{tabular}

Primary cells that had been stored in liquid nitrogen were recultured and transduced with SFV/hGM-CSF with a MOI of 5. Two days after transduction, the medium was harvested and hGM-CSF concentrations were determined. Untransduced cells served as controls to determine the baseline level of GM-CSF production. Transduction increased GM-CSF production to approximately 55 ng/10 6 cells. ND, not determined.

virus titers can be achieved that are comparable to yields obtained with the adenovirus systems. In addition, SFV can infect a broad range of target cell types. Although some members of the alphavirus genus are known to cause human disease, laboratory strains of SFV are considered to be nonpathogenic to humans. ${ }^{21}$ Moreover, the recombinant SFV system was derived from an attenuated strain. ${ }^{21}$ Alphaviruses are genetically relatively simple with a genome of approximately $10 \mathrm{~kb}$. Recombinant RNA consists of four genes together encoding the viral replicase and the transgene. Genes encoding the structural SFV proteins are not expressed by the recombinant SFV particles thereby minimizing immune responses against the viral vector. Although neutralizing antibodies have been described after multiple inoculations of macaques with SFV particles, titers were much lower than those arising against the transgene. ${ }^{26}$ pSFV1 was also used to express both subunits of human IL-12. ${ }^{38}$ Direct intratumoral injection of these SFV/IL-12 particles in mice significantly inhibited growth of established B16 melanomas and inhibited neovascularization. ${ }^{39}$

Recently, the application of alphavirus replicons has been extended to naked nucleic acids. ${ }^{40}$ The relatively high efficacy of self-replicating nucleic acids as compared with DNA vaccines applied in anticancer therapies was correlated with the apoptosis-inducing features of the RNA replicase $\mathrm{e}^{41-43}$ and was attributed to mimicking of a viral infection that may trigger danger signals. ${ }^{44}$ In contrast to SFV particles, injected nucleic acids are distributed more easily through the body of experimental animals and persist much longer. ${ }^{45}$ However, their persistence is a contraindication for expression of the apoptosis-inducing RNA replicase. A comparison of the efficacy of recombinant SFV particle immunization with DNA or RNA immunization encoding the same antigen showed that SFV particles induce stronger immune responses. ${ }^{45}$

Here, we set out to investigate the potential of the recombinant SFV system for the production of GM-CSFsecreting, autologous tumor cell vaccines. These vaccines can be used, for instance, as adjuvant therapies for the treatment of patients at high risk of metastases after the primary tumor is surgically removed. The advantage of this approach over, for instance, vaccination with tumor-specific proteins or peptides, is that an autologous tumor cell vaccine contains the complete repertoire of tumor-specific antigens which are present in the tumor. Additionally, such a vaccine has a patient-specific tumor antigen signature overcoming the problem of interpatient tumor antigen heterogeneity.

The results reported in this paper indicate the potential of the recombinant SFV system. We have cloned the GMCSF genes successfully into the recombinant SFV system. Using the Helper-2 system high-titer particle stocks could be obtained which encode biologically active GM-CSF. By performing ELISA it was demonstrated that high expression levels of the transgene are achieved using the SFV system. The levels obtained $24 \mathrm{~h}$ after transfection of the BHK-21 cell line with SFV/mGM-CSF vary between 2000 and $3500 \mathrm{ng}$ per $10^{6}$ cells (see Figure 1) which is relatively high (higher levels have only been described for recombinant vaccinia virus ${ }^{40}$ ). After transduction of BHK-21 cells with SFV/hGM-CSF, levels of hGM-CSF were obtained that varied between 400 and $500 \mathrm{ng}$ per $10^{6}$ cells after $24 \mathrm{~h}$. This cytokine production is more than 10 -fold the threshold level of $36 \mathrm{ng}$ per $10^{6}$ cells (after $24 \mathrm{~h}$ ), which was determined as the minimal immunologically active dose in preclinical trials. ${ }^{1}$

The GM-CSF levels obtained after transduction of B16F10 and the C26 cell lines are lower (Figure 1b and c) than when BHK-21 cells are used. With the retroviral system described by Dranoff, $300 \mathrm{ng} / \mathrm{ml}$ per $10^{6} \mathrm{~B} 16 \mathrm{~F} 10$ cells after $24 \mathrm{~h}$ could be obtained., ${ }^{70}$ However, also for the retroviral system expression levels vary from cell line to cell line. In C26 cells, the evaluation of $\beta$-galactosidase activity $24 \mathrm{~h}$ after transduction with SFV/LacZ particles was difficult; possibly these cells are more sensitive to alphavirus-induced apoptosis than the other cell lines used. ${ }^{20}$

In the primary cultures of human RCC samples murine GM-CSF expression levels were comparable or higher than the GM-CSF levels reported in the literature in similar samples transduced with retroviral/hGM-CSF-particles. ${ }^{1,8}$ However, expression of human GM-CSF by RCC was lower than murine GM-CSF but above the level of $35 \mathrm{ng} / 10^{6}$ cells which has been reported to be required to evoke an immune response in animal models. ${ }^{1}$ For BHK cells as well as renal cells we found that transduction with virus expressing human or murine GM-CSF resulted in an approximately five-fold higher production of the latter. This difference may be ascribed to differences in stability of the proteins and/or differences of the RNA molecules (eg codon usage and stability). The importance of a high expression level has not yet been established. Previously, mGM-CSF dosage effects have been described by Abe et al. ${ }^{12}$ These authors reported that 
adenoviral mGM-CSF vaccines expressing 20-fold higher mGM-CSF levels than retroviral mGM-CSF vaccines did not protect against tumor challenge, in contrast to the retroviral mGM-CSF vaccines. After diluting the adenoviral mGM-CSF vaccines 20-fold with untransduced cells, the adenoviral mGM-CSF vaccines showed the same efficacy as the retroviral vaccines. These results suggest a certain, yet to date unidentified, optimal mGM-CSF dose. In 1995, Schmidt et $a l^{46}$ described a similar IL-2 dosage effect. The findings may have considerable consequences for clinical application of cytokine-transduced, autologous tumor cell vaccines but also for production of the vaccines. Their results suggest that only a fraction of the vaccine cells needs to be transduced for an effective immune response. Moreover, not only the total amount of synthesized GM-CSF may influence vaccination efficacy, also the number of cells involved in cytokine production may influence efficacy. It has been suggested that smaller numbers of high IL-2-producing cells may be more efficacious than higher numbers of low-producing cells. ${ }^{47}$

Furthermore, we demonstrate that the vaccine preparations, including the tumor samples of human origin, produce GM-CSF for several days after transduction despite the process of apoptosis that is induced in SFVinfected cells. However, apoptosis is not induced immediately, allowing the native virus to replicate efficiently. In the vector system based on SFV, transgene expression proceeds for several days before protein synthesis stops. Thus, the SFV-prepared vaccines will produce GM-CSF at the site of vaccination for several days, which is likely to be essential for attracting immune effector cells. In addition, apoptosis of tumor cells presumably enhances the immune response against the tumor since apoptotic cells are taken up by antigen-presenting cells. In this process of cross-presentation these antigenpresenting cells will process the tumor antigens contributing to the efficient priming of specific cytotoxic $\mathrm{T}$ lymphocytes.

Recently, new recombinant alphavirus systems were developed with features that facilitate cloning of recombinant genes, ${ }^{48}$ improve heterologous protein production ${ }^{17}$ and improve biosafety of the system. ${ }^{18}$ Taken together, the characteristics of the recombinant SFV system described by others and in this paper indicate that this system provides a valuable new approach to deliver cytokine genes for the generation of autologous tumor cell vaccines.

\section{Materials and methods}

\section{Cell lines}

Baby hamster kidney (BHK-21) cells and mouse C26 colon cancer cells were cultured in GMEM (Gibco-BRL, Life Technologies, Breda, The Netherlands) containing 5\% fetal calf serum (FCS; PAA Laboratories, Linz, Austria). Mouse B16F10 cells (kindly provided by Dr M Schreurs, Department of Tumorimmunology, University of Nijmegen, The Netherlands) were cultured in RPMI 1640 (Gibco-BRL) supplemented with 10\% FCS. Vero cells (ATCC, CCL81) and BLK cells (ATCC, TIB-81) were cultured in DMEM (Gibco-BRL) supplemented with 10\% FCS. The human bladder tumor cell line RT112 ${ }^{49}$ was cultured in HAM/F12 medium supplemented with $10 \%$
FCS, using an atmosphere of $3 \% \mathrm{O}_{2}, 5 \% \mathrm{CO}_{2}$ and $92 \%$ $\mathrm{N}_{2}$. The lung epithelial TC-1 cells were derived from C57BL/ 6 mice and were cultured in IMDM (Gibco-BRL). Mouse FDCP-1 and human TF-1 cells were cultured in RPMI 1640 supplemented with 10\% FCS, 1\% glutamine and $10 \mathrm{ng} / \mathrm{ml}$ GM-CSF. All media contained $100 \mathrm{U} / \mathrm{ml}$ penicillin and $100 \mu \mathrm{g} / \mathrm{ml}$ streptomycin (Gibco-BRL).

\section{Renal cell carcinoma samples}

Only samples of histopathologically proven renal cell carcinomas (RCC) were used in this study. Each malignant tissue sample was accompanied with healthy kidney tissue from the same patient. All specimens were minced and collagenase type II (Bayer Diagnostics, Leverkusen, Germany; $500 \mathrm{U} / \mathrm{ml}$ RPMI1640 medium) and DNase (Sigma, Zwijndrecht, The Netherlands; $22 \mathrm{kU} / \mathrm{ml}$ RPMI1640 medium) were added 1:1 until the tumor material was entirely submerged. The tissue sample was placed at $37^{\circ} \mathrm{C}, 5 \% \mathrm{CO}_{2}$ for minimally $2 \mathrm{~h}$ or longer (maximally overnight) depending on the consistency of the tissue. The cells were washed twice in $10 \mathrm{ml}$ Hank's balanced salts solution (HBSS, Gibco-BRL) resuspended in RPMI1640 and cultured overnight at $37^{\circ} \mathrm{C}, 5 \% \mathrm{CO}_{2}$. For transduction purposes the cells were harvested and plated at the same density as the cell lines (see below).

\section{Plasmids}

pSFV1, pSFV-Helper2 and pSFV3/LacZ were kindly provided by Dr P Liljeström (Karolinska Institute, Stockholm, Sweden). Dr H Davis (Loeb Research Institute, Ottawa Civic Hospital, Canada) kindly provided the plasmid pRJB-GM carrying the murine GM-CSF gene, described by Altmann et al. ${ }^{50}$ Dr H Spits (The Netherlands Cancer Institute, Amsterdam, The Netherlands) kindly provided the plasmid pcDhuGM-CSF, carrying the human GM-CSF gene.

\section{Cloning of the mGM-CSF and hGM-CSF genes in pSFV1}

The forward primer: 5'-GAGGGATCCGCACCATGT GGCTGCAGA-3' (first three nucleotides are miscellaneous, followed by a BamHI site, a ribosomal binding site, and part of the GM-CSF sequence) and the backward primer: 5'-GAGGGATCCTGGATTCAGAGCTG-3' were used to amplify mGM-CSF (see GenBank accession number X03019 for details). For hGM-CSF, the forward primer: 5'-GAGGGATCCGCACCATGTGGCTGCAGAG CCT-3' and the backward primer: 5'-GAGGGAT CCTCATCTGGCCGGTCTCAC-3' were used. For the development of an optimal primer pair the last primer was paired to a sequence of noncoding DNA following the stop codon which was present in the plasmid (see GenBank accession number M10663). The mGM-CSF and hGM-CSF PCR products were isolated from agarose gel, restricted with BamHI, ligated into the BamHI site of pSFV1 and checked for correct orientation by restriction analysis. The resulting plasmids, pSFV1/mGM-CSF and pSFV1/hGM-CSF were sequenced on an automatic sequencer (ALF, Pharmacia, Roosendaal, The Netherlands).

Sequencing of the mGM-CSF insert in pSFV1 revealed a point mutation at nucleotide position 102, when compared with the sequence described in GenBank (accession number X03019). This mutation causes an Ile-Val amino acid change. The influence of this mutation was judged 
to be minor considering the structures of both amino acids and the fact that the point mutation is not located in one of the regions described to be critical for mGMCSF activity. ${ }^{51}$ It was decided to proceed with particle production and to examine whether the point mutation had any effect on the biological activity of the mGM-CSF encoded by SFV. The hGM-CSF gene present in pSFV1/hGM-CSF displayed no differences when compared with the GenBank sequence.

\section{Production of recombinant SFV particles}

pSFV-Helper-2, pSFV3/LacZ, pSFV1/mGM-CSF and pSFV1/hGM-CSF plasmids were isolated using the protocol accompanying the Qiagen (Hilden, Germany) midi plasmid purification kit and were restricted with SpeI (Gibco-BRL). RNA was synthesized using the MEGAscript (SP6) in vitro transcription kit (Ambion, Sanbio, Uden, The Netherlands). Routinely, $\pm 70 \mu \mathrm{g}$ of RNA was obtained per reaction starting from $1 \mu \mathrm{g}$ of template plasmid.

BHK-21 cells were plated at a density of $2 \times 10^{6}$ cells $/ 15 \mathrm{ml}$ medium and cultured as described. Two days later the cells were harvested and resuspended in phosphate buffered saline (PBS without calcium or magnesium; Gibco-BRL) at a concentration of $10 \times 10^{6}$ cells per $\mathrm{ml}$. Fifteen $\mu \mathrm{g}$ recombinant RNA and $7.5 \mu \mathrm{g}$ Helper 2 RNA (molecular ratio \pm 1:1) were admixed and electroporated into $800 \mu \mathrm{l}$ cell suspension $\left(=8 \times 10^{6} \mathrm{BHK}-21\right.$ cells $)$ using a Biorad Gene Pulser II (two pulses of $850 \mathrm{~V} / 25$ $\mu F)$ and Biorad electroporation cuvettes $(0.4 \mathrm{~cm})$. Routinely, time constants between 0.34 and 0.38 were observed. After pulsing, the cells were resuspended in $10 \mathrm{ml}$ GMEM medium and cultured for 24-36 h. Cellular debris was removed by centrifugation at $700 \mathrm{~g}$ in a 15 $\mathrm{ml}$ tube for $5 \mathrm{~min}$. The supernatant containing the viral particles was aliquoted, quick frozen in liquid nitrogen and stored at $-80^{\circ} \mathrm{C}$.

\section{Transduction with recombinant SFV particles}

The Helper-2 system ${ }^{15}$ carries a point mutation in a spike precursor protein which renders the spike fusion-inactive. Despite the point mutation the spike protein is incorporated into the virion but needs to be activated (cleaved) in vitro by $\alpha$-chymotrypsin treatment (conditionally infective). Recombinant SFV particles were activated by adding $1 / 20$ volume $\alpha$-chymotrypsin $(10 \mathrm{mg} / \mathrm{ml}$; Fluka Chemie, Sigma-Aldrich Chemie, Zwijndrecht, The Netherlands) for $30 \mathrm{~min}$ at room temperature (RT) and $\alpha$-chymotrypsin was inactivated by adding 1 volume aprotinin (Sigma; $2 \mathrm{mg} / \mathrm{ml}$ ). Cells from cell lines or from patient material were plated $5 \times 10^{4}$ cells per well $(500$ $\mu l)$ in 24 well-plates 1 day before transduction. The next day $300 \mu \mathrm{l}$ medium was removed and $200 \mu \mathrm{l}$ particle solution was added per well. After $1 \mathrm{~h} 200 \mu \mathrm{l}$ medium was added and cells were incubated overnight at $37^{\circ} \mathrm{C}, 5 \%$ $\mathrm{CO}_{2}$

\section{Titer determination of recombinant particle batches}

Titers of SFV/LacZ particles were determined by standard $\beta$-galactosidase detection techniques. Medium was removed and cells were fixed with $0.5 \mathrm{ml}$ fixation solution (2\% paraformaldehyde, $0.2 \%$ gluteraldehyde) for 5 min RT. Fixed cells were washed three times with PBS and stained with $0.5 \mathrm{ml}$ coloring solution $(0.1 \% \mathrm{X}$-gal in $\left.5 \mathrm{~mm} \mathrm{~K}_{3} \mathrm{Fe}(\mathrm{CN})_{6}, 5 \mathrm{~mm} \mathrm{~K}{ }_{4} \mathrm{Fe}(\mathrm{CN})_{6}, 2 \mathrm{~mm} \mathrm{MgCl}_{2}\right)$ at $37^{\circ} \mathrm{C}$.
Coloring was stopped after $\pm 2 \mathrm{~h}$ by removing the coloring solution and washing the cells with PBS. Color was preserved by adding $1 \mathrm{ml} 87 \%$ glycerol to the wells. Titers of SFV/mGM-CSF and SFV/hGM-CSF particle batches were routinely determined on BHK-21 cells by immunohistochemistry using a rabbit polyclonal antibody (kindly provided by Dr Tero Ahola, University of Helsinki, Finland) against SFV nsP3. For these purposes BHK-21 cells were plated at 50000 cells per well on an 8-well chamber slide (Nunc, Naperville, IL, USA) and the next day transduced with serial dilutions of SFV particles. On the third day, the cells were washed three times with PBS (containing 1\% bovine serum albumin) and fixed for $1 \mathrm{~min}$ in acetone at RT. PBS-moistened fixed slides were incubated with $200 \mu \mathrm{l}$ of rabbit anti-nsP3 (1:500 in PBS) solution for 30 min. Thereafter the slides were washed three times with PBS and incubated with $200 \mu \mathrm{l}$ secondary antibody (FITC anti-rabbit, 1:200). FITC fluorescing cells were detected by microscopy and counted. Titers were determined considering the virus dilution used in the specific well which was counted and expressed as the number of infecting units per $\mathrm{ml}$.

\section{Detection of GM-CSF in culture medium by ELISA}

GM-CSF levels were determined by ELISA analysis. The hGM-CSF ELISA was performed at the University Hospital. For both, hGM-CSF analysis and mGM-CSF analysis standard sandwich ELISAs were used. The antibodies used were commercially obtained from Pharmingen (ITK Diagnostics, Uithoorn, The Netherlands). The ELISAs were performed according to the protocol supplied by the manufacturer. Recombinant mGM-CSF produced by Escherichia coli (PeproTech, SanverTECH, Heerhugowaard, The Netherlands) and recombinant hGM-CSF (Novartis, Basel, Switzerland) were used as standards.

\section{Determination of GM-CSF biological activity}

The activity of mGM-CSF detected in the medium of transduced cells was determined using FDCP-1 cells, which are dependent on IL-3 or GM-CSF for their survival. FDCP-1 cells were harvested and IL-3 was removed by washing three times with PBS. Five thousand or 10000 FDCP-1 cells were plated $(100 \mu \mathrm{l}$ in DMEM $+10 \%$ FCS in a 96-well plate) per well. We first determined the growth curve of FDCP-1 cells in the presence of commercially obtained GM-CSF (PeproTech; Figure 2, open squares). FDCP-1 cell survival was determined after a 3-day culture, using the WST-1 cell proliferation assay (Boehringer Mannheim, Almere, The Netherlands). Next, on the basis of this curve and the GM-CSF concentration in the supernatant of SFV / mGMCSF transduced BHK cells, FDCP-1 cells were cultured in the presence of increasing volumes of this supernatant (volumes ranging between 0.01 to $2 \mu \mathrm{l}$ per $\mathrm{ml}$ culture medium).

The biological activity of hGM-CSF was determined using TF-1 cells. TF-1 cells are cytokine-dependent cells of human origin. The cells were cultured for five successive passages (split ratio 1:5) in the presence or absence of hGM-CSF. The number of cells was determined at each cell passage (recombinant rhGM-CSF as a control). In addition, primary human bone marrow cells were cultured in the presence or absence of hGM-CSF. Human bone marrow cells were obtained with informed consent from patients undergoing thorax surgery and cultured as 
described by Durkin et al..$^{52} 10^{5}$ Cells were incubated with either a standard control mixture of rhGM-CSF (10 $\mathrm{ng} / \mathrm{ml})$ and rhIL-3 (10 ng/ml), SFV-produced hGM-CSF (10 $\mathrm{ng} / \mathrm{ml})$, or without GM-CSF and IL-3.

\section{Acknowledgements}

The authors would like to thank P Liljeström and P Berglund for expert advice and valuable suggestions. In addition, we thank A Heikema, R Timmer, M Esselink and J Bijzet for their assistance with cloning, preparation of SFV batches and performing ELISAs. This work was funded by a grant from the Dutch Cancer Foundation (NKB/KWF).

\section{References}

1 Simons JW et al. Bioactivity of autologous irradiated renal cell carcinoma vaccines generated by ex vivo granulocyte-macrophage colony-stimulating factor gene transfer. Cancer Res 1997; 57: 1537-1546.

2 Abdel-Wahab Z et al. A phase I clinical trial of immunotherapy with interferon-gamma gene modified autologous melanoma cells: monitoring the humoral immune response. Cancer 1997; 80: $401-412$

3 Lotze MT et al. Cytokine gene therapy of cancer using interleukin-12: murine and clinical trials. Ann NY Acad Sci 1996; 795: 440-454.

4 Soiffer R et al. Vaccination with irradiated autologous melanoma cells engineered to secrete human granulocyte-macrophage colony-stimulating factor generates potent antitumor immunity in patients with metastatic melanoma. Proc Natl Acad Sci USA 1998; 95: 13141-13146.

5 Chang AE et al. Immunogenetic therapy of human melanoma utilizing autologous tumor cells transduced to secrete granulocyte-macrophage colony-stimulating factor. Hum Gene Ther 2000; 11: 839-850.

6 Mastrangelo MJ et al. Intratumoral recombinant GM-CSF-encoding virus as gene therapy in patients with cutaneous melanoma. Cancer Gene Ther 1999; 6: 409-422.

7 Dranoff G et al. Vaccination with irradiated tumor cells engineered to secrete murine granulocyte-macrophage colony-stimulating factor stimulates potent, specific, and long-lasting antitumor immunity. Proc Natl Acad Sci USA 1993; 90: 3539-3543.

8 Jaffee EM et al. High efficiency gene transfer into primary human tumor explants without cell selection. Cancer Res 1993; 53: $2221-2226$.

9 Sampson JH et al. Subcutaneous vaccination with irradiated, cytokine-producing tumor cells stimulates CD8(+) cell-mediated immunity against tumors located in the immunologically privileged' central nervous system. Proc Natl Acad Sci USA 1996; 93: 10399-10404.

10 Yu JS, Burwick JA, Dranoff G, Breakafield XO. Gene therapy for metastatic brain tumors by vaccination with granulocytemacrophage colony-stimulating factor-transduced tumor cells. Hum Gene Ther 1997; 8: 1065-1072.

11 Simons JW et al. Induction of immunity to prostate cancer antigens: results of a clinical trial of vaccination with irradiated autologous prostate tumor cells engineered to secrete granulocyte-macrophage colony-stimulating factor using ex vivo gene transfer. Cancer Res 1999; 59: 5160-5168.

12 Abe J et al. Antitumor effect induced by granulocyte/ macrophage colony-stimulating factor gene-modified tumor vaccination: comparison of adenovirus- and retrovirusmediated genetic transduction. J Cancer Res Clin Oncol 1995; 121: 587-592.

13 Chen $\mathrm{SH}$ et al. Combination suicide and cytokine gene therapy for hepatic metastases of colon carcinoma: sustained antitumor immunity prolongs animal survival. Cancer Res 1996; 56: 37583762.
14 Qin HX, Chatterjee SK. Construction of recombinant vaccinia virus expressing GM-CSF and its use as tumor vaccine. Gene Therapy 1996; 3: 59-66.

15 Berglund $\mathrm{P}$ et al. Semliki Forest virus expression system: production of conditionally infectious recombinant particles. Bio/Technology 1993; 11: 916-920.

16 Liljeström P, Garoff H. A new generation of animal cell expression vectors based on the Semliki Forest virus replicon. Bio/Technology 1991; 9: 1356-1361.

17 Sjöberg EM, Suomalainen M, Garoff H. A significantly improved Semliki Forest virus expression system based on translation enhancer segments from the viral capsid gene. Bio/Technology 1994; 12: 1127-1131.

18 Smerdou C, Liljeström P. Two-helper RNA system for production of recombinant Semliki Forest virus particles. J Virol 1999; 73: 1092-1098.

19 Strauss JH, Strauss EG. The alphaviruses: gene expression, replication, and evolution. Microbiol Rev 1994; 58: 491-562.

20 Griffin DE, Hardwick JM. Regulators on the road to persistent alphavirus infection. Ann Rev Microbiol 1997; 51: 565-592.

21 Atkins GJ, Sheahan BJ, Liljestrom P. The molecular pathogenesis of Semliki Forest virus: a model virus made useful? J Gen Virol 1999; 80: 2287-2297.

22 Daemen $\mathrm{T}$ et al. Genetic immunization against cervical carcinoma:induction of cytotoxic $\mathrm{T}$ lymphocyte activity with a recombinant alphavirus vector expressing human papilomavirus type 16 E6 and E7. Gene Therapy 2000; 7: 1859-1866.

23 Zhou $X$ et al. Generation of cytotoxic and humoral immune responses by non-replicative recombinant Semliki Forest virus. Proc Natl Acad Sci USA 1995; 92: 3009-3013.

24 Fleeton M, Liljestrom P, Sheahan B, Atkins G. Recombinant Semliki Forest virus particles expressing louping ill virus antigen induce better protective response than plasmid-based DNA vaccines or an inactivated whole particle vaccine. J Gen Virol 2000; 81: 749-758.

25 Calmenero P, Liljestrom P, Jondal M. Induction of P815 tumor immunity by recombinant Semliki Forest virus expressing the P1A gene. Gene Therapy 1999; 6: 1728-1733.

26 Mossman SP et al. Protection against lethal simian immunodeficiency virus SIVsmmPBj14 disease by a recombinant Semliki Forest virus gp160 vaccine and by a gp120 subunit vaccine. J Virol 1996; 70: 1953-1960.

27 Berglund $\mathrm{P}$ et al. Outcome of immunization of cynomolgus monkeys with recombinant Semliki Forest virus encoding human immunodeficiency virus type I envelope protein and challenge with a high dose of SHIV-4 virus. Aids Res Hum Retrovir 1997; 13: 1487-1495.

28 Klimp $\mathrm{AH}$ et al. Effect of intraperitoneally administered recombinant murine granulocyte-macrophage colony-stimulating factor (rmGM-CSF) on the cytotoxic potential of murine peritoneal cells. Br I Cancer 1999; 79: 89-94.

29 Klimp AH et al. Activation of peritoneal cells upon in vivo transfection with a recombinant alphavirus expressing GM-CSF. Gene Therapy 2001; 8: 300-307.

30 Keith WN, Brown R, Pragnell IB. Retrovirus mediated transfer and expression of GM-CSF in haematopoietic cells. $\mathrm{Br}$ J Cancer 1990; 62: 388-394.

31 Dranoff G. Strategies for cancer gene therapy. Cancer Chemother Biol Respone Modif 1997; 17: 328-348.

32 Dranoff G. Interpreting cancer vaccine clinical trials. J Gene Med 1999; 1: 80-83.

33 Jaffee EM. Immunotherapy of cancer. Ann NY Acad Sci 1999; 886: $67-72$.

34 Van Tendeloo VF, Van Broeckhoven C, Berneman ZN. Genebased cancer vaccines: an ex vivo approach. Leukemia 2001; 15: 545-558.

35 Esche C, Shurin MR, Lotze MT. The use of dendritic cells for cancer vaccination. Curr Opin Mol Ther 1999; 1: 72-81.

36 Parmiani G, Rodolfo M, Melani C. Immunological gene therapy with ex vivo gene-modified tumor cells: a critique and a reappraisal. Hum Gene Ther 2000; 11: 1269-1275. 
37 Berns AJ et al. Phase I study of non-replicating autologous tumor cell injections using cells prepared with or without GM-CSF gene transduction in patients with metastatic renal cell carcinoma. Hum Gene Ther 1995; 6: 347-368.

38 Zhang $\mathrm{J}$ et al. Cloning of human IL-12 p40 and p35 DNA into the Semliki Forest virus vector: expression of IL-12 in human tumor cells. Gene Therapy 1997; 4: 367-374.

39 Asselin-Paturel C et al. Transfer of the murine interleukin-12 gene in vivo by a Semliki Forest virus vector induces B16 tumor regression through inhibition of tumor blood vessel formation monitored by Doppler ultrasonography. Gene Therapy 1999; 6: 606-615.

40 Berglund $\mathrm{P}$ et al. Enhancing immune responses using suicidal DNA vaccines. Nat Biotechnol 1998; 16: 562-565.

41 Glasgow GM, McGee MM, Sheahan BJ, Atkins GJ. Death mechanisms in cultured cells infected by Semliki Forest virus. J Gen Virol 1997; 78: 1559-1563.

42 Ying $\mathrm{H}$ et al. Cancer therapy using a self-replicating RNA vaccine. Nat Med 1999; 5: 823-827.

43 Leitner WW et al. Enhancement of tumor-specific immune response with plasmid DNA replicon vectors. Cancer Res 2000; 60: $51-55$

44 Restifo NP, Ying H, Hwang L, Leitner WW. The promise of nucleic acid vaccines. Gene Therapy 2000; 7: 89-92.

45 Morris-Downes MM et al. Semliki Forest virus-based vaccines: persistence, distribution and pathological analysis in two animal systems. Vaccine 2001; 19: 1978-1988.

46 Schmidt W et al. Cancer vaccines: the interleukin 2 dosage effect. Proc Natl Acad Sci USA 1995; 92: 4711-4714.

47 Bonnekoh B et al. Ex vivo and in vivo adenovirus-mediated gene therapy strategies induce a systemic anti-tumor immune defence in the B16 melanoma model. J Invest Dermatol 1998; 110: 867-871.

48 Meanger J, Peroulis I, Mills J. Modified Semliki Forest virus expression vector that facilitates cloning. BioTechniques 1997; 23: 432-436.

49 Peacock JH et al. Initial DNA damage or repair as the major determinant of cellular radiosensitivity? Int J Radiat Biol 1989; 56: 543-547

50 Altman SW, Johnson GD, Prystowsky MB. Single proline substitutions in predicted $\alpha$-helices of murine granulocyte-macrophage colony-stimulating factor result in a loss in bioactivity and altered glycosylation. J Biol Chem 1991; 266: 5333-5341.

51 Shanafelt AB, Miyajima A, Kitamura T, Kastelein RA. The amino-terminal helix of GM-CSF and IL-5 governs high affinity binding to their receptors. EMBO J 1991; 10: 4105-4112.

52 Durkin JP et al. The identification and characterization of a novel human differentiation-inhibiting protein that selectively blocks erythroid differentiation. Blood 1992; 5: 1161-1171. 